\title{
Fault-Tolerant Metric Dimension of Generalized Wheels and Convex Polytopes
}

\author{
Zhi-Bo Zheng, ${ }^{1}$ Ashfaq Ahmad, ${ }^{2}$ Zaffar Hussain, ${ }^{3}$ Mobeen Munir $\left(D,{ }^{4}\right.$ \\ Muhammad Imran Qureshi $\mathbb{D}^{5},{ }^{5}$ Imtiaz Ali, ${ }^{6}$ and Jia-Bao Liu ${ }^{7}{ }^{7}$ \\ ${ }^{1}$ Department of Mathematics, Baoshan University, Baoshan 678000, China \\ ${ }^{2}$ Department of Computer Science, COMSATS University Islamabad, Lahore Campus, Defense Road Off Raiwind Road, \\ Lahore 54000, Pakistan \\ ${ }^{3}$ Department of Mathematics, The University of Lahore, Lahore 54500, Pakistan \\ ${ }^{4}$ Department of Mathematics, University of the Punjab, New Campus, Lahore 54590, Pakistan \\ ${ }^{5}$ Department of Mathematics, COMSATS University Islamabad, Vehari campus, Vehari 61100, Pakistan \\ ${ }^{6}$ Department of Mathematics, NCBA and E DHA Lahore, Lahore, Pakistan \\ ${ }^{7}$ School of Mathematics and Physics, Anhui Jianzhu University, Hefei 230601, China \\ Correspondence should be addressed to Mobeen Munir; mmunir@ue.edu.pk
}

Received 19 May 2020; Revised 12 August 2020; Accepted 18 September 2020; Published 4 November 2020

Academic Editor: Jean Jacques Loiseau

Copyright (C) 2020 Zhi-Bo Zheng et al. This is an open access article distributed under the Creative Commons Attribution License, which permits unrestricted use, distribution, and reproduction in any medium, provided the original work is properly cited.

For a graph $G$, an ordered set $S \subseteq V(G)$ is called the resolving set of $G$, if the vector of distances to the vertices in $S$ is distinct for every $v \in V(G)$. The minimum cardinality of $S$ is termed as the metric dimension of $G$. $S$ is called a fault-tolerant resolving set (FTRS) for $G$, if $S \backslash\{v\}$ is still the resolving set $\forall v \in V(G)$. The minimum cardinality of such a set is the fault-tolerant metric dimension (FTMD) of $G$. Due to enormous application in science such as mathematics and computer, the notion of the resolving set is being widely studied. In the present article, we focus on determining the FTMD of a generalized wheel graph. Moreover, a formula is developed for FTMD of a wheel and generalized wheels. Recently, some bounds of the FTMD of some of the convex polytopes have been computed, but here we come up with the exact values of the FTMD of two families of convex polytopes denoted as $D_{k}$ for $k \geq 4$ and $Q_{k}$ for $k \geq 6$. We prove that these families of convex polytopes have constant FTMD. This brings us to pose a natural open problem about the existence of a polytope having nonconstant FTMD.

\section{Introduction}

Computer networks and other similar systems can be given design of graphs in which vertices are the representor of nodes and edges as a medium of connection between them. The location of fault in the computer network is the vertex of a graph. This idea to identify each vertex in the network was initially given by Slater in [1] and then by Harary and Melter in [2] to control the fault in a better way. Therefore, the concepts of locating numbers and locating sets were developed. In fact, the actual terminology appeared way back by Blumenthal [3] for standard Euclidean spaces. However, not many researchers were forced to think about these ideas because of linear continuum nature of these spaces.
The resolving set and ideas, pretty similar to it, are contributing in diverse areas today merely because of their usage in discrete nature. These ideas are utilized to recognize nodes in a network, connected joins in graphs, mastermind games [4], strategies for the network discovery, combinatorial optimization, applications of pattern recognition, pharmaceutical chemistry, image processing, and game theory. A collection of points can be identified by finding the distance from all nodes in a network or graph. Thus, the problem of robot navigation may be solved by finding a minimal set of fixed vertices. Such minimal sufficiently large set is a resolving set of the graph. The corresponding cardinality is termed as metric dimension. 
For a connected, simple graph $G$, metric $d_{G}: V(G) \times$ $V(G) \longrightarrow \mathbb{N} \cup\{0\}$ is the distance between $x$ and $y$ and is denoted by $d_{G}(x, y)$. Let $W=\left\{w_{1}, w_{2}, \cdots, w_{k}\right\} \subseteq V(G)$ be an ordered set, and let $v \in V(G)$. Then, $r(v \mid W)$ is the representation of $v$ with respect to $W$ and is the $k$-tuple $\left(d\left(v, w_{1}\right), d\left(v, w_{2}\right), \cdots, d\left(v, w_{k}\right)\right)$. If $r(v \mid W)$ are distinct for distinct vertices of $G$, then we say $W$ is a resolving set for $G$, see [5]. A resolving set with minimum cardinality is a basis of $G$, and its cardinality is termed as the metric dimension of $G$, denoted by $\beta(G)$.

Buczkowski et al. computed the metric dimension of wheel $W_{n}$ and proved that $\lfloor 2 n+2 / 5\rfloor$ for $n \geq 7$ [5]. In [6], Hernando et al. proved that for fan, the metric dimension is $\lfloor 2 n+2 / 5\rfloor$ for $n \geq 7$, and for Jahangir graphs $J_{2 n}$, Tomescu and Javaid proved that $\lfloor 2 n / 3\rfloor$ for all $n \geq 4$, see [7]. In $[8,9]$, the authors proved some results about the metric dimension of regular graphs. In [10], the metric dimension for a flower graph is computed by the authors. In [11], Chartrand et al. proved that a metric dimension is 1 iff graph is a path. In 2014, the authors in [12] computed the metric dimension for the generalized wheel graph. Ali et al. [13], in 2012, and Munir et al. [14], in 2017, studied about the metric dimension of Möbius ladders. For unicyclic graphs, Poisson and Zhang found metric dimension in [15]. If for a family $\left(G_{n}\right)_{n \geq 1}$ of connected graphs, where exists a constant $C>0$ such that $\beta\left(G_{n}\right) \leq C$ for all $n \geq 1$, then the family of graphs is said to have bounded metric dimension; otherwise, it has unbounded metric dimension. If all graphs of a family have the same metric dimension (means do not depend on $n$ ), then that family is called a family with constant metric dimension [16]. For further study, one can see [17-26].

Recent advancement in the concept of metric dimension has paved a path for a new related idea known as fault tolerance. Suppose a network consists of $n$ processing units. To form a fault-tolerant self-stable system, it is essential that if any single unit becomes fail, another chain of units (excluding the faulty unit) can replace the originally used chain. So, the design of this fault-tolerance empowers the system to continue working, possibly at a reduced level and opposed to flop totally.

A resolving set $S$ is contemplate to be fault-tolerant if $S \backslash\{v\}$ is also a resolving set, $\forall v \in S$, and consequently, the minimum cardinality of such $S$ is termed as FTMD, denoted by $\beta^{\prime}(G)$. If the order of $S$ is equal to $\beta^{\prime}(G)$, then it is called a fault-tolerant metric basis. FTMD is constant for a family $\mathscr{G}$ of connected graphs if it is not dependent on any choice of the member of that family. Fault-tolerant designs are successfully used in computer sciences and engineering [27]. The study of fault-tolerant sets was inaugurated by Slater in [28]. Hernando et al. developed the concept of an FTMD in [29]. They studied the FTMD of trees. They also proved a very important result in the form of upper bound irrespective of the choice of graph which is given as $\beta^{\prime}(G) \leq \beta(G)\left(1+2 \cdot 5^{\beta(G)-1}\right)$. Javaid et. al. in $[30,31]$ studied fault-tolerance in resolvability, and also, they computed FTMD of few classes of graphs. It is not difficult to conclude that $\beta^{\prime}(G) \geq \beta(G)+1$. Few more results were found by
Shabbir and Zamfirescu in [32] about triangular lattice networks.

In this article, we considered families of convex polytopes and studied the FTMD for them. A convex polytope is the convex-hull of an finite set in any Euclidean space. These are significant graphs and hence have been under discussion from many years. For instance, Baca in $[33,34]$ computed labellings and magic of convex polytopes. Nazeer et al. computed the formulae for the average eccentricity, eccentricity, center, and periphery for the convex polytopes in [35]. In [21], Imran et al. found that the metric dimension is constant for convex polytopes $S_{k}, T_{k}, Q_{k}$, and $D_{k}$. In this article, we take a step further and prove that $D_{k}$ and $Q_{k}$ have constant FTMD. We also discuss the FTMD of multilevel wheel and classical wheel graphs.

\section{Main Results}

In this section, we put together our main results. At first, we prove that FTMD of $D_{k}$ is 4 . Then, we move towards the case of $Q_{k}$. In both cases, we use double inequality of sets. In the end, we compute the FTMD of multilevel wheel.

2.1. Fault-Tolerant Metric Dimension of the Graph of $D_{k}$ and $Q_{k}$. Let $A_{k}$ denote the antiprism graph. The graph $A_{k}$ is obtained using two regular $k$-gones say $P$ and $P$ with the vertices $\left\{u_{1}, u_{2}, \cdots, u_{k}\right\}$ and $\left\{z_{1}, z_{2}, \cdots, z_{k}\right\}$, respectively, in anticlockwise. We join any vertex $u_{p}$ of $P$ with the vertices $z_{p}$ and $z_{p+1}$ of $P$ by edges for $p=1,2, \cdots, k$. The graph of the convex polytope $D_{k}$ is formed [33], by the dual graph of $A_{k}$ consisting of the vertices $u_{1}, u_{2}, u_{3}, \cdots, u_{k}, x_{1}, x_{2}, x_{3}, \cdots$, $x_{k} ; y_{1}, y_{2}, y_{3}, \cdots, y_{k}$; and $z_{1}, z_{2}, z_{3}, \cdots, z_{k}$. It consists of 5 sided and $k$-sided faces. For compete details, see Figure 1.

The convex polytope $Q_{k}$ defined in [34] has faces of 3 sides, 4 sides, 5 sides, and $n$-sides. The vertex set for the graph of the convex polytope $Q_{k}$ is the same as that of the convex polytope $D_{k}$, and also, $E\left(Q_{k}\right)=E\left(D_{k}\right) \cup\left\{x_{p} x_{p+1}: 1\right.$ $\leq p \leq k\}$.

Let us call the vertices $u_{p}, 1 \leq p \leq k, x_{p}, 1 \leq p \leq k$, $y_{p}, 1 \leq p \leq k$, and $z_{p}, 1 \leq p \leq k$ as the inner vertices, the middle vertices, the interior vertices, and the outer vertices, respectively. The cycles induced by $\left\{u_{p}, 1 \leq p \leq k\right\}$, $\left\{x_{p}, 1 \leq p \leq k\right\}$, and $\left\{z_{p}, 1 \leq p \leq k\right\}$ are the inner cycle, the middle cycle, and the outer cycle respectively, as shown in Figure 2.

Theorem 1. For both type of convex polytopes $D_{k}$ and $Q_{k}$, $\beta^{\prime}=4$ for $k \geq 6$, where $k$ is an integer.

Proof

Case I: $k$ is even: let $k=2 n ; n \in Z^{+}$, and $n \geq 3$. Let $F=\left\{u_{1}, u_{2}, u n+1, u_{n+2}\right\}$. We shall prove that $F$ is a fault-tolerant metric basis for $D_{k}$ and $Q_{k}$ in the present case. We prove that the metric representations of all vertices of $D_{k}$ and $Q_{k}$ with respect to $F$ are distinct in at least two coordinates. We call $u_{k+1}, x_{k+1}, y_{k+1}$, and $z_{k+1}$ as the vertices of inner cycle, the inner vertices, the 


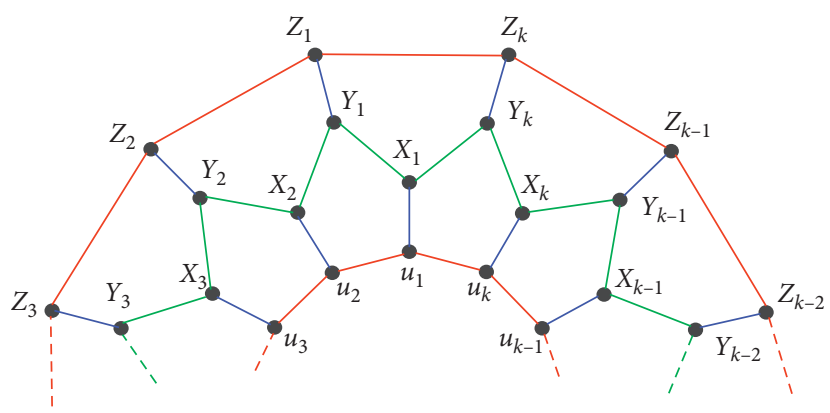

FIGURE 1: Graph of $D_{k}$.

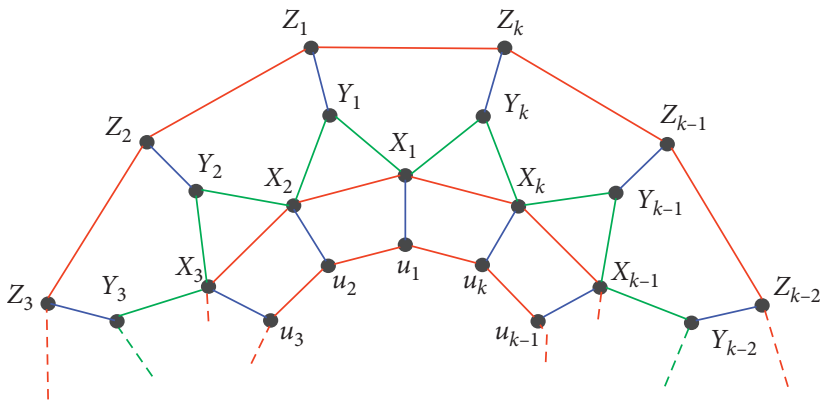

Figure 2: Graph of $Q_{k}$.

outer vertices, and the vertices of outer cycle, respectively.

The metric representations of vertices lying on the inner cycle are

$$
r\left(u_{p} \mid F\right)= \begin{cases}(-1+p,-2+p, n+1-p, n+2-p), & \text { if } 3 \leq p \leq n, \\ (2 n+1-p, 2 n+2-p, p-1-n, p-2-n), & \text { if } n+3 \leq p \leq 2 n .\end{cases}
$$

The metric representations of vertices belonging to inner vertices are

$$
r\left(x_{p} \mid F\right)= \begin{cases}(1,2, n+1, n), & \text { if } n=1, \\ (p,-1+p, n+2-p, n+3-p), & \text { if } 2 \leq p \leq n+1, \\ (2 n+2-p, 2 n+3-p, p-n, p-1-n), & \text { if } n+2 \leq p \leq 2 n\end{cases}
$$

The metric representations of vertices belonging to outer vertices are

$$
r\left(y_{p} \mid F\right)= \begin{cases}(2,2, n+1, n+1), & \text { if } p=1, \\ (p+1, p, n+2-p, n+3-p), & \text { if } 2 \leq p \leq n, \\ (n+1, n+1,2,2), & \text { if } p=n+1, \\ (2 n+2-p, 2 n+3-p, p+1-n, p-n), & \text { if } n+2 \leq p \leq 2 n .\end{cases}
$$


The metric representations of vertices lying on the outer cycle are

$$
r\left(z_{p} \mid F\right)= \begin{cases}(3,3,2+n, 2+n), & \text { if } p=1, \\ (2+p, 1+p, 3+n-p, 4+n-p), & \text { if } 2 \leq p \leq n, \\ (n+2, n+2,3,3), & \text { if } p=n+1, \\ (2 n+3-p, 2 n-p+4, p-n+2, p-n+1), & \text { if } n+2 \leq p \leq 2 n .\end{cases}
$$

These representations are distinct in at least two coordinates. So, $F$ is an FTRS. Therefore, for both $\left(D_{k}\right)$ and $\left(Q_{k}\right), \beta^{\prime} \leq 4$. Since for both $\left(D_{k}\right)$ and $\left(Q_{k}\right), \beta=3$ as proved in [21], so $\beta^{\prime}>3$. Hence, $\beta^{\prime}=4$ when $k$ is even. Case II: $k$ is an odd integer: let $k=2 n+1$ where $n \in Z^{+}$ and $n \geq 3$, and $F=\left\{u_{1}, u_{2}, u_{n+1}, u_{n+2}\right\}$. We shall prove that $F$ is a fault-tolerant metric basis for $D_{k}$ and $Q_{k}$ in the present case. We prove that the metric representations of all vertices of $D_{k}$ and $Q_{k}$ with respect to $F$ are distinct in at least two coordinates.

The metric representations of vertices lying on the inner cycle are

$$
r\left(u_{p} \mid F\right)= \begin{cases}(-1+p,-2+p, 1+n-p, 2+n-p), & \text { if } 3 \leq p \leq n, \\ (2 n+2-p, 2 n+3-p, p-n-1, p-n-2), & \text { if } n+3 \leq p \leq 2 n+1\end{cases}
$$

The metric representations of inner vertices are

$$
r\left(x_{p} \mid F\right)= \begin{cases}(1,2, n+1, n+1), & \text { if } p=1, \\ (p,-1+p, 2+n-p, 3+n-p), & \text { if } 2 \leq p \leq n+1, \\ (n+1, n+1,2,1), & \text { if } p=n+2, \\ (2 n-p+3,2 n-p+4, p-n, p-n-1), & \text { if } n+3 \leq p \leq 2 n+1 .\end{cases}
$$

The metric representations of outer vertices are

$$
r\left(y_{p} \mid F\right)= \begin{cases}(2,2, n+1, n+2), & \text { if } p=1, \\ (p+1, p, n-p+2, n-p+3), & \text { if } 2 \leq p \leq n, \\ (n+2, n+1,2,2), & \text { if } p=n+1, \\ (2 n-p+3,2 n-p+4, p-n+1, p-n), & \text { if } n+2 \leq p \leq 2 n+1 .\end{cases}
$$

The metric representations of vertices on the outer cycle are

$$
r\left(z_{p} \mid F\right)= \begin{cases}(3,3,2+n, n+3), & \text { if } p=1, \\ (2+p, 1+p, n+3-p, n+4-p), & \text { if } 2 \leq p \leq n, \\ (n+3, n+2,3,3), & \text { if } p=n+1, \\ (2 n-p+3,2 n-p+4, p-n+2, p-n+1), & \text { if } n+2 \leq p \leq 2 n+1 .\end{cases}
$$


These representations are distinct in at least two coordinates. So, $F$ is an FTRS. Therefore, for both $\left(D_{k}\right)$ and $\left(Q_{k}\right)$, $\beta^{\prime} \leq 4$. Since for both $\left(D_{k}\right)$ and $\left(Q_{k}\right), \beta=3$ as proved in [21], so $\beta^{\prime}>3$. Hence, $\beta^{\prime}=4$ when $k$ is odd.

\subsection{Fault Tolerance in Metric Dimension of Multilevel Wheel.} In this part, we focus on the FTMD of multilevel wheel $\left(W_{n, m}\right)$. An $m$ wheel graph of size $n$ is defined as follows: an $m$ wheel graph of size $n$ can be composed of $m C_{n}+K_{1}$. It consists of $m$ cycles of size $n$, where the vertices of the $m$ cycles are all connected to a common hub. Thus, $W_{n, m}$ has $n m+1$ vertices, the center, and $n$ rim vertices and has diameter 2. Figure 3 represents the graph of $W_{8, m}$.

A classical wheel graph is simply a specialized wheel with a single level denoted as $W_{n, 1}$ or simply $W_{n}$. Here, we give the graph of $W_{8,1}$ in Figure 4.

We do not include the central vertex in the representation of the above graph $W_{8,1}$. In the above graph, the vertices of the graph $G$ are $V(G)=\left\{v_{1}, v_{2}, v_{3}, v_{4}, v_{5}, v_{6}, v_{7}, v_{8}\right\}$. Now, consider the resolving sets $R_{1}=\left\{v_{2}, v_{4}, v_{6}\right\}, R_{2}=\left\{v_{2}, v_{6}, v_{8}\right\}, R_{3}=$ $\left\{v_{2}, v_{4}, v_{8}\right\}$, and $R_{4}=\left\{v_{4}, v_{6}, v_{8}\right\}$. For $R_{1}=\left\{v_{2}, v_{4}, v_{6}\right\}$, it is easy to see that $r\left(v_{1} / R_{1}\right)=(1,2,2), r\left(v_{2} / R_{1}\right)=(0,2,2), r\left(v_{3} /\right.$ $\left.R_{1}\right)=(1,1,2), r\left(v_{4} / R_{1}\right)=(2,0,2), r\left(v_{5} / R_{1}\right)=(2,1,1), r\left(v_{6} /\right.$ $\left.R_{1}\right)=(2,2,0), r\left(v_{7} / R_{1}\right)=(2,2,1)$, and $r\left(v_{8} / R_{1}\right)=(2,2,2)$. Since the vertices of $G$ have distinct representations with respect to $R_{1}, R_{1}$ is a resolving set. Since metric dimension of a wheel graph $W_{8}$ is $3, R_{1}$ is a basis for $G$ because it has metric dimension 3. Similarly, $R_{2}, R_{3}$, and $R_{4}$ are also basis for $G$ with metric dimension 3. Now, $R_{5}=R_{1} \cup R_{2} \cup R_{3} \cup R_{4}$ or $R_{5}=\left\{v_{2}\right.$, $\left.v_{4}, v_{6}, v_{8}\right\}$.

We see that $R_{5} \backslash\{v\}$ is also a resolving set for each $v$ in $R_{5}$. As $\beta\left(W_{8,1}\right)$ of the above example is 3 , therefore, $\beta\left(W_{8,1}\right)=4$. Hence, $R_{5}$ is a fault-tolerant metric basis with $\beta^{\prime}\left(W_{8,1}\right)=4$, so $R_{5}$ is an FTMS.

If $B$ is a resolving set of the graph $G$, which contains two or more vertices of $G$, and we suppose that the vertices of $B=\left\{v_{j_{1}}, v_{j_{2}}, \cdots, v_{j_{r}}\right\}$, so the $j_{1}<j_{2}<\cdots<j_{r}$. We shall say that the vertices $v_{j_{a}}, v_{j_{a}}+1$ for $i=1,2, \cdots, r-1$, and $v_{j_{r}}, v_{j_{1}}$ are neighboring vertices. Now, the vertices lying between these neighboring vertices are referred to as the gap of $B$ and the number of vertices in a gap will be referred to as the size of the gap. If $|B|$ is $t$, then it has $t$ gapes, and some of which may be empty. For example, the gap between $v_{2}$ and $v_{4}$ in Figure 4 is 1 because there is only one vertex $v_{3}$ in between $v_{2}$ and $v_{4}$. Now, let $B$ is an FTMS of $\left(W_{n, m}\right)$. If we do not include the central vertex, then we make the following two conditions:

(1) If $n$ is even, then every gap of $B$ contains exactly one vertex. If a gap contains zero vertex, then $B \backslash\{v\}$ does not remain as an FTMS. Similarly, if a gap contains two or more vertices, then again $B \backslash\{v\}$ does not remain as an FTMS, which is a contradiction.

(2) If $n$ is odd, then every gap of $B$ contains exactly one vertex except for one gap which contains zero vertex. If more than one gap contains zero vertex, then $B \backslash\{v\}$ does not remain as an FTMS. Similarly, if a gap

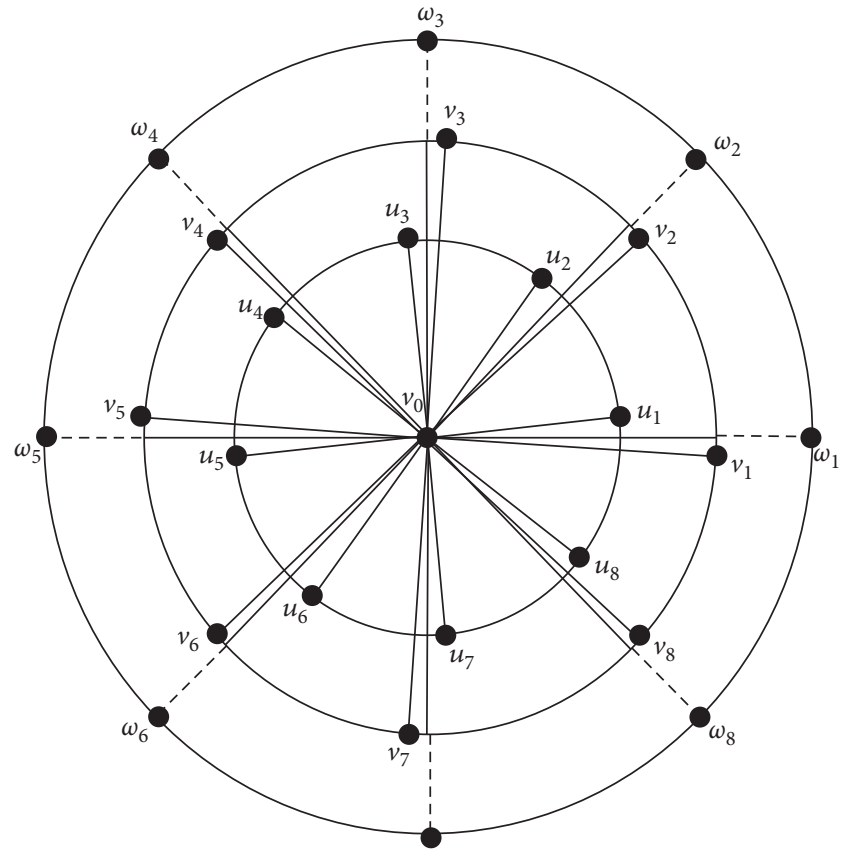

Figure 3: Graph of $W_{8, m}$.

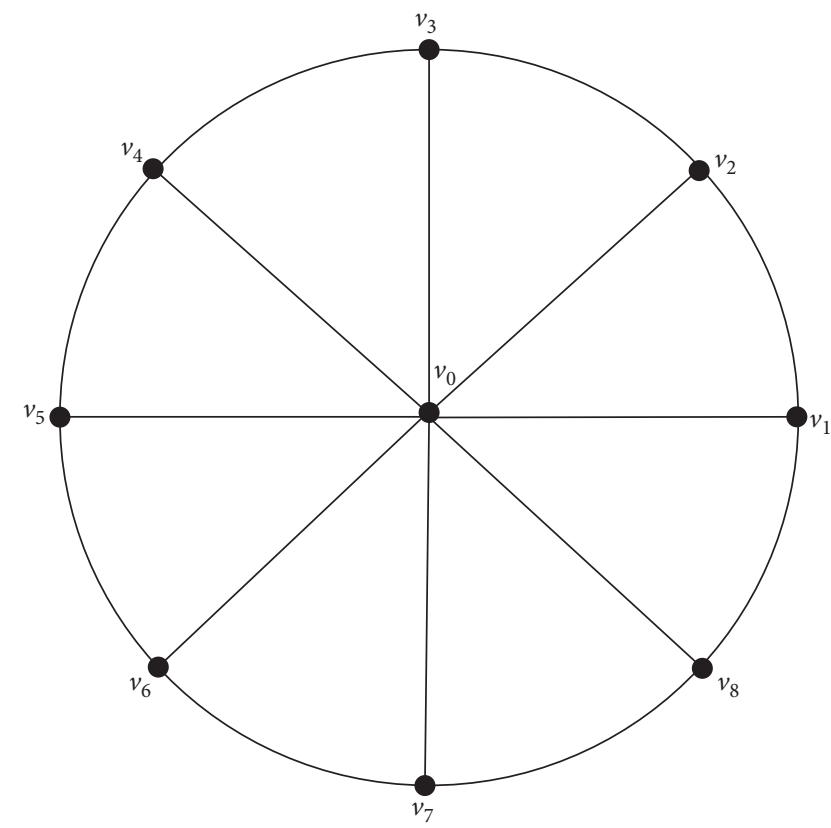

Figure 4: A graph of $W_{(8,1)}$ wheel.

contains two or more vertices, then again $B \backslash\{v\}$ does not remain as an FTMS, which is a contradiction.

Our aim in this section is to compute the FTMD of wheel $W_{n}$ and generalize the wheel graph $W_{n, r}$. In [5], it was shown that $\operatorname{dim}\left(W_{n}\right)=\lceil(2 n+5) / 2\rceil$, for every $n \geq 7$. Now, suppose that $B^{\prime}$ is any fault-tolerant metric basis of $W_{n}$. We observe the following results in the form of lemmas and theorems. 


\subsubsection{Lemmas}

Lemma 1. If $n$ is even and $n \geq 8$, then every gap of $B^{\prime}$ (faulttolerant basis) contains exactly one vertex.

Proof.

(i) Suppose on the contrary, there exists a gap which contains two vertices and at least one of its neighboring gaps contains one vertex. Then, $\exists v_{i}, v_{i+1}, v_{i+2}, v_{i+3}, v_{j}$ (where $v_{j}$ is neighboring vertex of $v_{i+3}$ and contains one vertex), such that $v_{i}, v_{i+3}, v_{j}$ belong to $B^{\prime}$. Then, by $B^{\prime} \backslash\left\{v_{i+3}\right\}$, we obtained $r\left(v_{i+2} / B^{\prime}\right)=r\left(v_{i+3} / B^{\prime}\right)=(2,2,2, \cdots, 2)$, which is a contradiction.

(ii) Now, again on the contrary, there exists a gap which contains two vertices and its neighboring gaps are empty. Then, there exist vertices $v_{i}, v_{i+1}, v_{i+2}, v_{i+3}$, $v_{i+4}, v_{i+5}, v_{j}$ (where $v_{j}$ is neighboring vertex of $v_{i+5}$ and is empty while another neighboring gap of $v_{i}$ is not empty), such that $v_{i}, v_{i+1}, v_{i+4}, v_{i+5}, v_{j}$ belong to $B^{\prime}$. Then, by $B^{\prime} \backslash\left\{v_{i}\right\}$, we have $r\left(v_{i} / B^{\prime}\right)=r\left(v_{i+2} /\right.$ $\left.B^{\prime}\right)=(1,2,2, \cdots, 2)$, which is a contradiction.

(iii) Now, again on the contrary, there exists a gap which contains two vertices and its neighboring gaps are empty. Then, there exist vertices $v_{i}, v_{i+1}$, $v_{i+2}, v_{i+3}, v_{i+4}, v_{i+5}, v_{i+6}, v_{i+7}$. Then, there exists $v_{j}$, any other vertex of $B^{\prime}$, such that $v_{i}, v_{i+1}, v_{i+2}$, $v_{i+5}, v_{i+6}, v_{i+7}, v_{j}$ belong to $B^{\prime}$. Then, by $B^{\prime} \backslash\left\{v_{j}\right\}$, we have $r\left(v_{n-1} / B^{\prime}\right)=r\left(v_{n-2} / B^{\prime}\right)=(2,2,2, \cdots, 2)$, which is a contradiction.

Lemma 2. If $n$ is odd and $n \geq 7$, then every gap of $B^{\prime}$ (faulttolerant basis) contains exactly one vertex except for one gap which is empty.

Proof. Suppose on the contrary, there exists a gap which contains two vertices. Then, $\exists v_{i}, v_{i+1}, v_{i+2}, v_{i+3}, v_{j}$ (where $v_{j}$ is neighboring vertex of $v_{i+3}$ ), such that $v_{i}, v_{i+3}, v_{j}$ belong to $B^{\prime}$. Then, by $B^{\prime} \backslash\left\{v_{j}\right\}$, we have $r\left(v_{i+2} / B^{\prime}\right)=r\left(v_{i+4} / B^{\prime}\right)$, which is a contradiction.

Lemma 3. The resolving set of $C_{n}$ of the type $B^{\prime}=\left\{v_{1}, v_{3}\right.$, $\left.v_{5}, \ldots \ldots v_{n-1}\right\}$ or $B^{\prime}=\left\{v_{2}, v_{4}, v_{6}, \ldots \ldots v_{n}\right\}$ is fault-tolerant metric basis.

Proof. We see from Lemmas 1 and 2 that $B^{\prime}$ can be an FTMS because for each $v$ in $B^{\prime}$, the set $B^{\prime} \backslash\{v\}$ is a resolving set. Now, we see that the set $B^{\prime} \backslash\left\{v_{1}, v_{3}\right\}$ or $B^{\prime} \backslash\left\{v_{2}, v_{4}\right\}$ is not a resolving set, which is a contradiction. Hence, $B^{\prime}$ is a fault-tolerant metric basis.

Lemma 4. If $C_{1}$ and $C_{2}$ denote the vertices of circle 1 and circle 2, respectively, then fault-tolerant metric basis for $C_{1}$ and $C_{2}$ is the same.

Proof. Let $C_{1}$ and $C_{2}$ denote the set of vertices of circle 1 and circle 2, respectively. Let $v_{i} \in C_{1}$ and $w_{i} \in C_{2}$. Now, $d\left(v_{i}, w_{i}\right)=d\left(w_{i}, v_{i}\right)=2$. Also, there does not exist any $v_{i}$ belongs to $C_{1}$ such that by $B^{\prime} \backslash\left\{v_{i}\right\}$, we get $r\left(v_{j} / B^{\prime}\right)=r\left(v_{r} / B^{\prime}\right)=(2,2,2, \cdots, 2)$. Similarly, there does not exist any $w_{i}$ belongs to $C_{2}$ such that by $B^{\prime} \backslash\left\{v_{i}\right\}$, we get $r\left(w_{j} / B^{\prime}\right)=r\left(w_{r} / B^{\prime}\right)=(2,2,2, \cdots, 2)$. In the same way, we can show that all levels have the same FTMS.

Now, we move towards the main results of this section.

Theorem 2. If $n \geq 7$ and $m \geq 1$, then we have $\beta\left(W_{n, m}\right)=$ $m\lceil n / 2\rceil$.

Proof. (when $m=1$ ).

We divide the proof into the following two cases based on parity of $n$.

(i) Case I:. when $n$ is even: if $n$ is even, then we can write $n=2 k+4$, where $k \geq 2$ and $\beta^{\prime}\left(W_{n, 1}\right)=\lfloor n / 2\rfloor=k+2$. Then, $B=\left\{V_{2 i}, 1 \leq i \leq n / 2\right\}$ is an FTMS as it satisfies conditions 1 and 2 .

(ii) Case II: when $n$ is odd: when $n$ is odd, then we may write $n=2 k+3$, where $k \geq 2$ and $\beta^{\prime}\left(W_{n, 1}\right)=\lceil n / 2\rceil=$ $k+2 . B=\left\{V_{1}, V_{2 i}, 1 \leq i \leq(n-1) / 2\right\}$. It is an FTMS as it satisfies conditions 1 and 2 . It follows from the above discussion that $\beta^{\prime}\left(W_{n, 1}\right)=\lceil n / 2\rceil$.

Next, we show that $\beta,\left(W_{n, 1}\right)=\lceil n / 2\rceil$ is the cardinality of FTMS. From Case 1 and Case 2, one can note that $B$ can be an FTMS because for each $v$ in $B$, the set $B \backslash\{v\}$ is a resolving set. Now, we show that $B$ is an FTMS. Suppose that $B$ is not an FTMS, then there exists vertex $v$ in $B$ such that $C=B \backslash\{v\}$ is an FTMS. Since $C$ is an FTMS as each $v$ in $C, C \backslash\{v\}$ should be a resolving set. However, $C \backslash\{v\}$ is not a resolving set which is a contradiction. Hence, $B$ is an FTMS.

Proof. (when $m=2$ ).

(i) Case I: when $n$ is even: when $n$ is even, then we may write $n=2 k+4$, where $k \geq 2$ and $\beta^{\prime}\left(W_{n, 2}\right)=2\lceil n /$ $2\rceil=2(k+2)$. Then, $B=\left\{v_{2 i}, w_{2 i}, 1 \leq i \leq n / 2\right\}$ is an FTMS as it satisfies conditions 1 and 2 .

(ii) Case II: when $n$ is odd: then, we may write $n=2 k+3$, where $k \geq 2$ and $\beta^{\prime}\left(W_{n, 2}\right)=2\lceil n / 2\rceil=2(k+2)$. Then, $B=\left\{v_{1}, v_{2 i}, w_{1}, w_{2 i}, 1 \leq i \leq(n-1) / 2\right\}$ is an FTMS as it satisfies both conditions 1 and 2 . It follows from the above discussion that $\beta^{\prime}\left(W_{n, 2}\right)=2\lceil n / 2\rceil$.

Next, we show that $\beta,\left(W_{n, 2}\right)=2\lceil n / 2\rceil$ is the cardinality of the FTMS. We see from Case 1 and Case 2 that $B$ can be an FTMS because for each $v$ in $B$, the set $B \backslash\{v\}$ is a resolving set. We show that $B$ is an FTMS. Suppose on the contrary that $B$ is not an FTMS, then there exists a $v$ in $B$ such that $C=B \backslash\{v\}$ is an FTMS. Now, $C$ is an FTMS. Therefore, for each $v$ in $C$, $C \backslash\{v\}$ should be a resolving set. However, $C \backslash\{v\}$ is not a resolving set which is a contradiction. Hence, $B$ is an FTMS.

Proof. (when $m \geq 3$ ). We shall prove this case by mathematical induction. 
TABLE 1: Comparison table for generalized wheel graphs.

\begin{tabular}{|c|c|c|c|c|c|c|}
\hline$n$ & $\beta^{\prime}\left(W_{n, 1}\right)$ & $\beta^{\prime}\left(W_{n, 2}\right)$ & $\beta^{\prime}\left(W_{n, m}\right)$ & $\beta^{\prime}\left(W_{n, 1}\right)$ & $\beta^{\prime}\left(W_{n, 2}\right)$ & $\beta^{\prime}\left(W_{n, m}\right)$ \\
\hline 7 & 3 & 7 & $3 m+2$ & 4 & 8 & $4 m$ \\
\hline 8 & 3 & 7 & $4 m-1$ & 4 & 8 & $4 m$ \\
\hline ? & 4. & 9 & $4 m$ & 5 & 10 & $5 m$ \\
\hline . & . & . & . & . & . & . \\
\hline · & $\cdot$ & $\cdot$ & $\cdot$ & $\cdot$ & $\cdot$ & $\cdot$ \\
\hline$n$ & $\left\lfloor\frac{2 n+2}{5}\right\rfloor$ & $\left\lfloor\frac{2 n+2}{5}\right\rfloor+\left\lceil\frac{2 n+4}{5}\right\rceil$ & $\left\lfloor\frac{2 n+2}{5}\right\rfloor+(m-1)\left\lfloor\frac{2 n+4}{5}\right\rfloor$ & $\left\lceil\frac{n}{2}\right\rceil$ & $2\left\lceil\frac{n}{2}\right\rceil$ & $m\left\lceil\frac{n}{2}\right\rceil$ \\
\hline
\end{tabular}

For level 1, we have $m=1$ and then $\beta^{\prime}\left(W_{n, 1}\right)=\lceil n / 2\rceil$. For level 2, i.e., $m=2$, we have $\beta^{\prime}\left(W_{n, 2}\right)=2\lceil n / 2\rceil$. Now, we suppose that the assertion is true for level $k$, i.e.,

$$
\beta^{\prime}\left(W_{n, k}\right)=k\lceil n / 2\rceil \cdot(1)
$$

We shall show that it is true for $m=k+1$. We have $\beta^{\prime}\left(W_{n, k+1}\right)=\beta^{\prime}\left(W_{n, k}\right)+\beta^{\prime}\left(W_{n, 1}\right)$.

Then,

$$
\begin{aligned}
& \beta^{\prime}\left(W_{n, k+1}\right)=k\left\lceil\frac{n}{2}\right\rceil+\left\lceil\frac{n}{2}\right\rceil, \\
& \beta^{\prime}\left(W_{n, k+1}\right)=(k+1)\left\lceil\frac{n}{2}\right\rceil .
\end{aligned}
$$
$m \geq 3$.

Hence, the given statement is true for all positive integers

\section{Examples}

3.1. Example 1. Now, we give some examples to compute the FTMD of the $E W_{(11,1)}$ wheel graph.

We see from Theorem 3 that the FTMD of $W_{(11,1)}$ is equal to 6 and the fault-tolerant metric basis for $E W_{(11,1)}$ is $B=\left\{v_{1}, v_{2}, v_{4}, v_{6}, v_{8}, v_{10}\right\}$. We see that $B=\left\{v_{1}, v_{4}, v_{6}, v_{10}\right\}$ is a metric basis for $W_{(11,1)}$ with cardinality 4 . In order to find fault-tolerant metric basis with cardinality 5 , we need other four metric bases having cardinality 4 other than $B=\left\{v_{1}, v_{4}, v_{6}, v_{10}\right\}$. However, $W_{(11,1)}$ has only one metric basis with cardinality 4 . It means that there is no faulttolerant metric basis with cardinality 5 . So, we need to add two vertices to find fault-tolerant metric basis. Hence, $B=$ $\left\{v_{1}, v_{2}, v_{4}, v_{6}, v_{8}, v_{10}\right\}$ is a fault-tolerant metric basis with cardinality 6 , and it also satisfies Lemma 1.

3.2. Example 2. We also give another example to compute the FTMD of the $E W_{(11,2)}$ wheel graph and verify.

We see from Theorem 4 that the FTMD of $E W_{(11,2)}$ is equal to 12 and the fault-tolerant metric basis for $E W_{(11,2)}$ is $B=\left\{v_{1}, v_{2}, v_{4}, v_{6}, v_{8}, v_{10}, w_{1}, w_{2}, w_{4}, w_{6}, w_{8}, w_{10}\right\}$. The FTMD for level $2(m=2)$ will be the same as that of level $1(m=1)$ because level 2 contains the same number of vertices as that of level 1. Hence, $B=\left\{v_{1}, v_{2}, v_{4}, v_{6}, v_{8}, v_{10}, w_{1}, w_{2}, w_{4}, w_{6}\right.$, $\left.w_{8}, w_{10}\right\}$ is a fault-tolerant metric basis with cardinality 12 , and it also satisfies Lemmas 1 and 2 .

\section{Conclusions and Open Problem}

In this article, we computed the FTMD of graphs of two convex polytopes denoted by $D_{n}$ and $Q_{n}$. We conclude that all these graphs have constant FTMD.

Theorem 3. The fault-tolerant metric dimension of convex polytopes $D_{n}$ is 4 for $n \geq 4$.

Theorem 4. The fault-tolerant metric dimension of convex polytopes $Q_{n}$ is 4 for $n \geq 6$.

We pose a natural open problem: Does there exist the graph of a convex polytope which has nonconstant FTMD? We also computed the FTMD of generalized wheel and arrive in the following theorem.

Theorem 5. If $n \geq 7$ and $m \geq 1$, then we have $\beta^{\prime}\left(W_{n, m}\right)=m\lceil n / 2\rceil$.

In Table 1, we presented the comparison between the metric dimension and fault-tolerant metric dimension for generalized wheel graphs.

\section{Data Availability}

No data were used to support the findings of this study.

\section{Conflicts of Interest}

The authors declare no conflicts of interest.

\section{Acknowledgments}

All the authors acknowledge their respective departments. This work was supported in part by the Joint Special Foundation on Basic Research in Local Colleges and Universities for the Department of Science and Technology of Yunnan Province of China under grant no. 2017FH001-106 and Quality Engineering for Rsearch Projects of the Anhui Department of Education about Wisdom classroom no. 2018zhkt180.

\section{References}

[1] P. J. Slater, "Leaves of trees," Congressus Numerantium in Proceeding of the 6th Southeastern Conference on Combinatorics, Graph Theory and Computing, vol. 14, pp. 549-559, 1975. 
[2] F. Harary and R. A. Melter, "On the metric dimension of a graph,” ARS Combinatoria, vol. 2, pp. 191-195, 1976.

[3] L. M. Blumenthal, Theory and Applications of Distance Geometry, Chelsea Publishing Company, Chelsea, VT, USA, 1970.

[4] V. Chvátal, "Mastermind," Combinatorica, vol. 3, no. 3-4, pp. 325-329, 1983.

[5] P. S. Buczkowski, G. Chartrand, C. Poisson, and P. Zhang, "On $k$-dimensional graphs and their bases," Periodica Mathematica Hungarica, vol. 46, no. 1, pp. 9-15, 2003.

[6] C. Hernando, M. Mora, I. M. Pelayo, C. Seara, J. Puertas, and C. Seara, "On the metric dimension of some families of graphs," Electronic Notes in Discrete Mathematics, vol. 22, pp. 129-133, 2005.

[7] I. Tomescu and I. Javaid, "On the metric dimension of the Jahangir graph," Bulletin mathématique de la Société des Sciences Mathématiques de Roumanie, vol. 50(98), no. 4, pp. 371-376, 2007.

[8] I. Javaid, M. T. Rahim, and K. Ali, "Families of regular graphs with constant metric dimension," Utilitas Mathematica, vol. 65, pp. 21-33, 2008.

[9] I. Tomescu and M. Imran, "On metric and partition dimensions of some infinite regular graphs," Bulletin mathématique de la Société des Sciences Mathématiques de Roumanie, vol. 52(100), no. 4, pp. 461-472, 2009.

[10] M. Imran, F. Bashir, A. Q. Baig, S. Bokhary, A. Riasat, and I. Tomescu, "On metric dimension of flower graphs $f_{n, m}$, and convex polytopes," Utilitas Mathematica, vol. 92, pp. 389-409, 2013.

[11] G. Chartrand, L. Eroh, M. A. Johnson, and O. R. Oellermann, "Resolvability in graphs and the metric dimension of a graph," Discrete Applied Mathematics, vol. 105, no. 1-3, pp. 99-113, 2000.

[12] H. M. A. Siddiqui and M. Imran, "Computing the metric dimension of wheel related graphs," Applied Mathematics and Computation, vol. 242, pp. 624-632, 2014.

[13] M. Ali, G. Ali, M. Imran et al., "On the metric dimension of Möbius ladders,” ARS Combinatoria, vol. 105, pp. 403-410, 2012.

[14] M. Munir, A. R. Nizami, Z. Iqbal, and H. Saeed, "On the metric dimension of Möbius ladder," ARS Combinatoria, vol. 135, pp. 249-256, 2017.

[15] C. Poisson and P. Zhang, "The metric dimension of unicyclic graphs," Journal of Combinatorial Mathematics and Combinatorial Computing, vol. 40, pp. 17-32, 2002.

[16] I. Tomescu and M. Imran, "Metric dimension and $R$-sets of connected graphs," Graphs and Combinatorics, vol. 27, no. 4, pp. 585-591, 2011.

[17] S. Khuller, B. Raghavachari, and A. Rosenfeld, "Location in graphs," Technical Report CS-TR-3326, University of Maryland, Colleg Park, MD, USA, 1994.

[18] S. Khuller, B. Raghavachari, and A. Rosenfeld, "Landmarks in graphs," Discrete Applied Mathematics, vol. 70, no. 3, pp. 217-229, 1996.

[19] R. A. Melter and I. Tomescu, "Metric bases in digital geometry," Computer Vision, Graphics, and Image Processing, vol. 25, no. 1, pp. 113-121, 1984.

[20] P. J. Slater, "Dominating and reference sets in graphs," Journal of Mathematical and Physical Sciences, vol. 22, no. 4, pp. 445-455, 1998

[21] M. Imran, A. Q. Baig, and A. Ahmed, "Families of plane graphs with constant metric dimension," Utilitas Mathematica, vol. 88, pp. 43-57, 2012.
[22] C. Gary and Z. Ping, "The theory and applications of resolvability in graphs: a survey," in Proceedings of the 34th Southeastern International Conference on Combinatorics, Graph Theory and Computing, pp. 47-68, Boca Raton, FL, USA, 2003.

[23] R. Naeem and M. Imran, "On resolvability and exchange property for resolving sets in antiweb-wheels," Utilitas Mathematica, vol. 104, pp. 187-200, 2017.

[24] M. Imran and H. M. A. Siddiqui, "Computing the metric dimension of convex polytopes generated by wheel related graphs," Acta Mathematica Hungarica, vol. 149, no. 1, pp. 10-30, 2016.

[25] M. Imran, S. A. u. H. Bokhary, A. Semaničová-feňovčíková, and A. Semanicova-Fenovcikova, "On classes of regular graphs with constant metric dimension," Acta Mathematica Scientia, vol. 33, no. 1, pp. 187-206, 2013.

[26] M. Basak, L. Saha, G. K. Das, and K. Tiwary, "Fault-tolerant metric dimension of circulant graphs $\mathrm{Cn}(1,2,3)$," Theoretical Computer Science, vol. 817, p. 66, 2020.

[27] J. P. Hayes, "A graph model for fault-tolerant computing systems," IEEE Transactions on Computers, vol. C-25, no. 9, pp. 875-884, 1976.

[28] P. J. Slater, "Fault-tolerant locating-dominating sets," Discrete Mathematics, vol. 249, no. 1-3, pp. 179-189, 2002.

[29] C. Hernando, M. Mora, P. J. Slater, and D. R. Wood, "On fault-tolerant metric dimension of graphs," Ramanujan Mathematical Society Lecture Notes, in Proceedings of the International Conference Convexity in Discrete Structures, vol. 5, pp. 81-85, 2008.

[30] I. Javaid, M. Salman, M. A. Chaudhry, and S. Shokat, "Faulttolerance in resolvability," Utilitas Mathematica, vol. 80, pp. 263-275, 2009.

[31] M. A. Chaudhry, I. Javaid, and M. Salman, "Fault-tolerant metric and partition dimension of graphs," Utilitas Mathematica, vol. 83, pp. 187-199, 2010.

[32] A. Shabbir and T. Zamfirescu, "Fault-tolerant designs in triangular lattice networks," Applicable Analysis and Discrete Mathematics, vol. 10, no. 2, pp. 447-456, 2016.

[33] M. Baca, "Labellings of two classes of convex polytopes," Utilitas Mathematica, vol. 34, pp. 24-31, 1988.

[34] M. Baca, "On magic labellings of convex polytopes," Annals of Discrete Mathematics, vol. 51, pp. 13-16, 1992.

[35] W. Nazeer, S. Kang, S. Nazeer et al., "On center, periphery and average eccentricity for the convex polytopes," Symmetry, vol. 8, no. 12, 2016. 